\title{
The infinite sum of the cubes of reciprocal Pell numbers
}

\section{Zhefeng Xu and Tingting Wang*}

"Correspondence:

tingtingwang126@126.com

Department of Mathematics,

Northwest University, Xi'an, Shaanxi,

P.R. China

\begin{abstract}
Given the sequence of Pell numbers $\left\{P_{n}\right\}$, we evaluate the integral part of the reciprocal of the sum $\sum_{k=n}^{\infty} \frac{1}{P_{n}^{3}}$ explicitly in terms of the Pell numbers themselves.

MSC: Primary 11B39
\end{abstract}

Keywords: Pell numbers; floor function; identity

\section{Introduction}

For any integer $n \geq 0$, the well-known Pell numbers $P_{n}$ are defined by the second-order linear recurrence sequence $P_{n+2}=2 P_{n+1}+P_{n}$, where $P_{0}=0$ and $P_{1}=1$. The Pell-Lucas numbers $Q_{n}$ are defined by $Q_{n+2}=2 Q_{n+1}+Q_{n}$, where $Q_{0}=2$ and $Q_{1}=2$. Let $\alpha=1+\sqrt{2}$ and $\beta=1-\sqrt{2}$. Then from the characteristic equations $x^{2}-2 x-1=0$, we also have the computational formulae

$$
P_{n}=\frac{1}{2 \sqrt{2}}\left(\alpha^{n}-\beta^{n}\right) \quad \text { and } \quad Q_{n}=\alpha^{n}+\beta^{n} .
$$

For example, the first few values of $P_{n}$ and $Q_{n}$ are $P_{0}=1, P_{1}=2, P_{2}=5, P_{3}=12, P_{4}=29, \ldots$, $Q_{0}=2, Q_{1}=2, Q_{2}=6, Q_{3}=14, Q_{4}=34, Q_{5}=82, \ldots$.

Various properties of the Pell numbers and related sequences have been studied by many authors, see [1-6]. For example, Santos and Sills [3] studied the arithmetic properties of the $q$-Pell sequence and obtained two identities. Kilic [4] studied the generalized order- $k$ Fibonacci-Pell sequences and gave several congruences. Recently, the authors [7] and [8] studied the infinite sums derived from the Pell numbers and proved the following identities:

$$
\begin{aligned}
& \left\lfloor\left(\sum_{k=n}^{\infty} \frac{1}{P_{k}}\right)^{-1}\right\rfloor= \begin{cases}P_{n-1}+P_{n-2} & \text { if } n \text { is even and } n \geq 2 ; \\
P_{n-1}+P_{n-2}-1 & \text { if } n \text { is odd and } n \geq 1,\end{cases} \\
& \left\lfloor\left(\sum_{k=n}^{\infty} \frac{1}{P_{k}^{2}}\right)^{-1}\right\rfloor= \begin{cases}2 P_{n-1} P_{n}-1 & \text { if } n \text { is an even number } \\
2 P_{n-1} P_{n} & \text { if } n \text { is an odd number, }\end{cases}
\end{aligned}
$$

where $\lfloor x\rfloor$ is the floor function, that is, it denotes the greatest integer less than or equal to $x$.

Some related works can also be found in [9] and [10]. Especially in [10], the authors studied a problem, which is little different from (1). That is, they studied the computa-

\section{Springer}

(c) $2013 \mathrm{Xu}$ and Wang; licensee Springer. This is an Open Access article distributed under the terms of the Creative Commons Attribution License (http://creativecommons.org/licenses/by/2.0), which permits unrestricted use, distribution, and reproduction in any medium, provided the original work is properly cited. 
tional problem of the nearest integer function of $\left(\sum_{k=n}^{\infty} \frac{1}{u_{k}}\right)^{-1}$ and proved an interesting conclusion:

$$
\left\|\left(\sum_{k=n}^{\infty} \frac{1}{u_{k}}\right)^{-1}\right\|=u_{n}-u_{n-1} \quad \text { for all } n>n_{0},
$$

where $\|\cdot\|$ denotes the nearest integer, namely $\|x\|=\left\lfloor x+\frac{1}{2}\right\rfloor,\left\{u_{n}\right\}_{n \geq 0}$ is an integer sequence satisfying the recurrence formula

$$
u_{n}=a u_{n-1}+u_{n-2}+\cdots+u_{n-s} \quad(s \geq 2)
$$

with the initial conditions $u_{0} \geq 0, u_{k} \in \mathbf{N}, 1 \leq k \leq s-1$.

Using the method in [10] seems to be very difficult to deal with $\left(\sum_{k=n}^{\infty} \frac{1}{u_{k}^{s}}\right)^{-1}$ for all integers $s \geq 2$.

The main purpose of this paper related to the computing problem of

$$
P(s, n) \equiv\left\lfloor\left(\sum_{k=n}^{\infty} \frac{1}{P_{k}^{s}}\right)^{-1}\right\rfloor
$$

for all integers $s \geq 3$. At the end of [7], the authors asked whether there exists a corresponding formula for $P(3, n)$.

In fact, this problem is difficult because it is quite unclear a priori what the shape of the result might be. In order to resolve the question, we carefully applied the method of undetermined coefficients and constructed a number of delicate inequalities in order to complete a proof. The result is as follows.

Theorem For any positive integer $n \geq 1$, we have the identity

$$
P(3, n)= \begin{cases}P_{n}^{2} P_{n-1}+3 P_{n} P_{n-1}^{2}+\left\lfloor-\frac{61}{82} P_{n}-\frac{91}{82} P_{n-1}\right\rfloor & \text { if } n \text { is even and } n \geq 2 \\ P_{n}^{2} P_{n-1}+3 P_{n} P_{n-1}^{2}+\left\lfloor\frac{61}{82} P_{n}+\frac{91}{82} P_{n-1}\right\rfloor & \text { if } n \text { is odd and } n \geq 1 .\end{cases}
$$

It remains a difficult problem even to conjecture what might be an analogous expression to the formula for $P(3, n)$ in the theorem for $P(k, n)$ when $k \geq 4$.

\section{Proof of the theorem}

In this section, we shall prove our theorem directly. First we consider the case that $n=2 m$ is an even number. It is clear that in this case our theorem is equivalent to

$$
\begin{aligned}
& P_{2 m}^{2} P_{2 m-1}+3 P_{2 m} P_{2 m-1}^{2}-\frac{1}{82}\left(61 P_{2 m}+91 P_{2 m-1}\right) \\
& \quad<\left(\sum_{k=2 m}^{\infty} \frac{1}{P_{k}^{3}}\right)^{-1}<P_{2 m}^{2} P_{2 m-1}+3 P_{2 m} P_{2 m-1}^{2}-\frac{1}{82}\left(61 P_{2 m}+91 P_{2 m-1}\right)+\frac{1}{82}
\end{aligned}
$$


or

$$
\begin{aligned}
& \frac{1}{P_{2 m}^{2} P_{2 m-1}+3 P_{2 m} P_{2 m-1}^{2}-\frac{1}{82}\left(61 P_{2 m}+91 P_{2 m-1}\right)+\frac{1}{82}} \\
& \quad<\sum_{k=2 m}^{\infty} \frac{1}{P_{k}^{3}}<\frac{1}{P_{2 m}^{2} P_{2 m-1}+3 P_{2 m} P_{2 m-1}^{2}-\frac{1}{82}\left(61 P_{2 m}+91 P_{2 m-1}\right)} .
\end{aligned}
$$

Now we prove that for all positive integers $k$, we have the inequality

$$
\begin{aligned}
\frac{1}{P_{2 k}^{3}}+\frac{1}{P_{2 k+1}^{3}}< & \frac{1}{P_{2 k}^{2} P_{2 k-1}+3 P_{2 k} P_{2 k-1}^{2}-\frac{1}{82}\left(61 P_{2 k}+91 P_{2 k-1}\right)} \\
& -\frac{1}{P_{2 k+2}^{2} P_{2 k+1}+3 P_{2 k+2} P_{2 k+1}^{2}-\frac{1}{82}\left(61 P_{2 k+2}+91 P_{2 k+1}\right)}
\end{aligned}
$$

It is clear that (3) holds for $k=1,2,3$ and 4 . So, without loss of generality, we can assume that $k \geq 5$. Note that $P_{2 k}^{3}=\frac{1}{8}\left(P_{6 k}-3 P_{2 k}\right), P_{2 k+1}^{3}=\frac{1}{8}\left(P_{6 k+3}+3 P_{2 k+1}\right), P_{2 k}^{3}+P_{2 k+1}^{3}=\frac{1}{8}\left(P_{6 k+3}+\right.$ $\left.P_{6 k}+3 P_{2 k+1}-3 P_{2 k}\right), P_{2 k}^{3} P_{2 k+1}^{3}=\frac{1}{512}\left(Q_{12 k+3}-6 Q_{8 k+2}+9 Q_{4 k+1}+4\right)$ and

$$
P_{2 k}^{2} P_{2 k-1}+3 P_{2 k} P_{2 k-1}^{2}=\frac{1}{8}\left(P_{6 k-1}+3 P_{6 k-2}+5 P_{2 k-1}+5 P_{2 k}\right),
$$

so inequality (3) is equivalent to

$$
\begin{aligned}
& \frac{8\left(P_{6 k+3}+P_{6 k}+3 P_{2 k+1}-3 P_{2 k}\right)}{Q_{12 k+3}-6 Q_{8 k+2}+9 Q_{4 k+1}+4} \\
& \quad<\frac{378 P_{6 k-1}+154 P_{6 k-2}-\frac{78}{41} P_{2 k+1}-\frac{318}{41} P_{2 k}}{\left(P_{6 k-1}+3 P_{6 k-2}-\frac{39}{41} P_{2 k}-\frac{159}{41} P_{2 k-1}\right)\left(P_{6 k+5}+3 P_{6 k+4}-\frac{39}{41} P_{2 k+2}-\frac{159}{41} P_{2 k+1}\right)}
\end{aligned}
$$

From the definition and properties of $P_{n}$ and $Q_{n}$, we can easily deduce the identities

$$
\begin{aligned}
& P_{n} P_{k}=\frac{1}{8} Q_{n+k}-\frac{(-1)^{k}}{8} Q_{n-k}, \quad n \geq k \\
& Q_{n} Q_{k}=Q_{n+k}+(-1)^{k} Q_{n-k}, \quad n \geq k \\
& P_{n} Q_{k}=P_{n+k}+(-1)^{k} P_{n-k}, \quad n \geq k .
\end{aligned}
$$

So, applying these formulae, we have

$$
\left(P_{6 k-1}+3 P_{6 k-2}\right)\left(P_{6 k+5}+3 P_{6 k+4}\right)=\frac{1}{8}\left(8 Q_{12 k+3}+10 Q_{12 k+2}-2,772\right)
$$

and

$$
\begin{aligned}
\left(P_{6 k-1}+3 P_{6 k-2}-\frac{39}{41} P_{2 k}-\frac{159}{41} P_{2 k-1}\right)\left(P_{6 k+5}+3 P_{6 k+4}-\frac{39}{41} P_{2 k+2}-\frac{159}{41} P_{2 k+1}\right) \\
=\frac{1}{8}\left(8 Q_{12 k+3}+10 Q_{12 k+2}-\frac{7,344}{41} Q_{8 k+1}-\frac{2,124}{41} Q_{8 k}-\frac{30,226,500}{1,681} Q_{4 k-3}\right) \\
\quad-\frac{1}{8}\left(\frac{12,507,840}{1,681} Q_{4 k-4}+\frac{4,442,760}{1,681}\right) .
\end{aligned}
$$


From these two identities and (4), we deduce that inequality (3) is equivalent to

$$
\begin{aligned}
& \frac{P_{6 k+3}+P_{6 k}+3 P_{2 k+1}-3 P_{2 k}}{Q_{12 k+3}-6 Q_{8 k+2}+9 Q_{4 k+1}+4} \\
& \quad<\frac{378 P_{6 k-1}+154 P_{6 k-2}-\frac{78}{41} P_{2 k+1}-\frac{318}{41} P_{2 k}}{8 Q_{12 k+3}+10 Q_{12 k+2}-\frac{7,344}{41} Q_{8 k+1}-\frac{2,124}{41} Q_{8 k}-\frac{30,226,500}{1,681} Q_{4 k-3}-\frac{12,507,840}{1,681} Q_{4 k-4}-\frac{4,442,760}{1,681}} .
\end{aligned}
$$

For convenience, we let

$$
\begin{aligned}
A= & \left(P_{6 k+3}+P_{6 k}+3 P_{2 k+1}-3 P_{2 k}\right) \times\left(8 Q_{12 k+3}+10 Q_{12 k+2}-\frac{7,344}{41} Q_{8 k+1}\right. \\
& \left.-\frac{2,124}{41} Q_{8 k}-\frac{30,226,500}{1,681} Q_{4 k-3}-\frac{12,507,840}{1,681} Q_{4 k-4}-\frac{4,442,760}{1,681}\right)
\end{aligned}
$$

and

$$
B=\left(Q_{12 k+3}-6 Q_{8 k+2}+9 Q_{4 k+1}+4\right)\left(378 P_{6 k-1}+154 P_{6 k-2}-\frac{78}{41} P_{2 k+1}-\frac{318}{41} P_{2 k}\right) .
$$

Then by calculation it follows that

$$
\begin{aligned}
A= & 8\left(P_{18 k+6}+P_{6 k}\right)+10\left(P_{18 k+5}+P_{6 k-1}\right)-\frac{7,344}{41}\left(P_{14 k+4}+P_{2 k-2}\right) \\
& -\frac{2,124}{41}\left(P_{14 k+3}+P_{2 k-3}\right)-\frac{30,226,500}{1,681}\left(P_{10 k}-P_{2 k+6}\right)-\frac{12,507,840}{1,681}\left(P_{10 k-1}\right. \\
& \left.+P_{2 k+7}\right)-\frac{4,442,760}{1,681} P_{6 k+3}+8\left(P_{18 k+3}-P_{6 k+3}\right)+10\left(P_{18 k+2}-P_{6 k+2}\right) \\
& -\frac{7,344}{41}\left(P_{14 k+1}-P_{2 k+1}\right)-\frac{2,124}{41}\left(P_{14 k}-P_{2 k}\right)-\frac{30,226,500}{1,681}\left(P_{10 k-3}-P_{2 k+3}\right) \\
& -\frac{12,507,840}{1,681}\left(P_{10 k-4}+P_{2 k+4}\right)-\frac{4,442,760}{1,681} P_{6 k}+3 \times 8\left(P_{14 k+4}+P_{10 k+2}\right) \\
& +3 \times 10\left(P_{14 k+3}+P_{10 k+1}\right)-\frac{7,344 \times 3}{41}\left(P_{10 k+2}+P_{6 k}\right)-\frac{2,124 \times 3}{41}\left(P_{10 k+1}\right. \\
& \left.+P_{6 k-1}\right)-\frac{30,226,500 \times 3}{1,681}\left(P_{6 k-2}+P_{2 k-4}\right)-\frac{12,507,840 \times 3}{1,681}\left(P_{6 k-3}+P_{2 k-5}\right) \\
& -\frac{4,442,760}{1,681} P_{2 k+1}-3 \times 8\left(P_{14 k+3}-P_{10 k+3}\right)-3 \times 10\left(P_{14 k+2}-P_{10 k+2}\right) \\
& +\frac{7,344 \times 3}{41}\left(P_{10 k+1}-P_{6 k+1}\right)+\frac{2,124 \times 3}{41}\left(P_{10 k}-P_{6 k}\right)+\frac{30,226,500 \times 3}{1,681}\left(P_{6 k-3}\right. \\
& \left.-P_{2 k-3}\right)+\frac{12,507,840 \times 3}{1,681}\left(P_{6 k-4}-P_{2 k-4}\right)+\frac{4,442,760}{1,681} P_{2 k} \\
= & 154 P_{18 k+3}+70 P_{18 k+2}-\frac{95,514}{41} P_{14 k+1}-\frac{38,910}{41} P_{14 k}-\frac{486,612,540}{1,681} P_{10 k-3} \\
& -\frac{201,554,538}{1,681} P_{10 k-4}-\frac{977,366,722}{1,681} P_{6 k-3}-\frac{344,423,038}{1,681} P_{6 k-4} \\
& -\frac{285,928,452}{1,681} P_{2 k-4}-\frac{118,454,868}{1,681} P_{2 k-5}, \\
B= & 378\left(P_{18 k+2}+P_{6 k+4}\right)+154\left(P_{18 k+1}-P_{6 k+5}\right)-\frac{78}{41}\left(P_{14 k+4}+P_{10 k+2}\right) \\
&
\end{aligned}
$$




$$
\begin{aligned}
& -\frac{318}{41}\left(P_{14 k+3}-P_{10 k+3}\right)-6 \times 378\left(P_{14 k+1}+P_{2 k+3}\right)-6 \times 154\left(P_{14 k}-P_{2 k+4}\right) \\
& +\frac{78 \times 6}{41}\left(P_{10 k+3}+P_{6 k+1}\right)+\frac{318 \times 6}{41}\left(P_{10 k+2}-P_{6 k+2}\right)+9 \times 378\left(P_{10 k}-P_{2 k-2}\right) \\
& +9 \times 154\left(P_{10 k-1}-P_{2 k-3}\right)-\frac{78 \times 9}{41}\left(P_{6 k+2}+P_{2 k}\right)-\frac{318 \times 9}{41}\left(P_{6 k+1}-P_{2 k+1}\right) \\
& +4 \times 378 P_{6 k-1}+4 \times 154 P_{6 k-2}-\frac{78 \times 4}{41} P_{2 k+1}-\frac{318 \times 4}{41} P_{2 k} \\
& =154 P_{18 k+3}+70 P_{18 k+2}-\frac{95,514}{41} P_{14 k+1}-\frac{38,910}{41} P_{14 k}+\frac{158,064}{41} P_{10 k} \\
& +\frac{64,416}{41} P_{10 k-1}+\frac{36,496}{41} P_{6 k-1}+\frac{14,880}{41} P_{6 k-2}-\frac{225,516}{41} P_{2 k-2}-\frac{92,796}{41} P_{2 k-3} .
\end{aligned}
$$

Observe that the major terms of $A$ and $B$ (above those of order $P_{10 k}$ ) are in total agreement. Note that $P_{n+2}=2 P_{n+1}+P_{n}$, we have

$$
\begin{aligned}
B-A= & \frac{41,028,234}{1,681} P_{10 k}+\frac{17,049,300}{1,681} P_{10 k-1} \\
& +\frac{348,025,790}{1,681} P_{6 k-2}+\frac{290,016,982}{1,681} P_{6 k-3} \\
& +\frac{39,772,560}{1,681} P_{2 k-2}+\frac{16,612,800}{1,681} P_{2 k-3}>0
\end{aligned}
$$

for all integers $k \geq 1$. So, inequalities (3), (4) and (5) hold for all integers $k \geq 1$.

Now, applying (3) repeatedly, we have

$$
\begin{aligned}
\sum_{k=2 m}^{\infty} \frac{1}{P_{k}^{3}}= & \sum_{k=m}^{\infty}\left(\frac{1}{P_{2 k}^{3}}+\frac{1}{P_{2 k+1}^{3}}\right) \\
< & \sum_{k=m}^{\infty} \frac{1}{P_{2 k}^{2} P_{2 k-1}+3 P_{2 k} P_{2 k-1}^{2}-\frac{1}{82}\left(61 P_{2 k}+91 P_{2 k-1}\right)} \\
& -\sum_{k=m}^{\infty} \frac{1}{P_{2 k+2}^{2} P_{2 k+1}+3 P_{2 k+2} P_{2 k+1}^{2}-\frac{1}{82}\left(61 P_{2 k+2}+91 P_{2 k+1}\right)} \\
= & \frac{1}{P_{2 m}^{2} P_{2 m-1}+3 P_{2 m} P_{2 m-1}^{2}-\frac{1}{82}\left(61 P_{2 m}+91 P_{2 m-1}\right)} .
\end{aligned}
$$

On the other hand, we prove the inequality

$$
\begin{aligned}
\frac{1}{P_{2 k}^{3}}+\frac{1}{P_{2 k+1}^{3}}> & \frac{1}{P_{2 k}^{2} P_{2 k-1}+3 P_{2 k} P_{2 k-1}^{2}-\frac{1}{82}\left(61 P_{2 k}+91 P_{2 k-1}\right)+\frac{1}{82}} \\
& -\frac{1}{P_{2 k+2}^{2} P_{2 k+1}+3 P_{2 k+2} P_{2 k+1}^{2}-\frac{1}{82}\left(61 P_{2 k+2}+91 P_{2 k+1}\right)+\frac{1}{82}} .
\end{aligned}
$$

This inequality is equivalent to

$$
\begin{aligned}
& \frac{P_{6 k+3}+P_{6 k}+3 P_{2 k+1}-3 P_{2 k}}{Q_{12 k+3}-6 Q_{8 k+2}+9 Q_{4 k+1}+4} \\
& >\frac{378 P_{6 k-1}+154 P_{6 k-2}-\frac{78}{41} P_{2 k+1}-\frac{318}{41} P_{2 k}}{\left(P_{6 k-1}+3 P_{6 k-2}-\frac{39}{41} P_{2 k}-\frac{159}{41} P_{2 k-1}+\frac{4}{41}\right)\left(P_{6 k+5}+3 P_{6 k+4}-\frac{39}{41} P_{2 k+2}-\frac{159}{41} P_{2 k+1}+\frac{4}{41}\right)}
\end{aligned}
$$


or

$$
\begin{aligned}
& \frac{4}{41}\left(P_{6 k+3}+P_{6 k}+3 P_{2 k+1}-3 P_{2 k}\right)\left(60 P_{6 k+1}+40 P_{6 k}-\frac{552}{41} P_{2 k}-\frac{396}{41} P_{2 k-1}+\frac{4}{41}\right) \\
& \quad>B-A
\end{aligned}
$$

or

$$
\begin{aligned}
& 140 Q_{12 k+3}+140 Q_{12 k+2}+\frac{1,200}{41} Q_{8 k+2}+\frac{5,952}{41} Q_{8 k+1}+\frac{19,116}{41} Q_{4 k}+\frac{9,444}{41} Q_{4 k-1} \\
& +\frac{4}{41} P_{6 k+3}+\frac{4}{41} P_{6 k}+\frac{12}{41} P_{2 k+1}-\frac{12}{41} P_{2 k}+\frac{47,728}{41}>\frac{41}{4}(B-A) .
\end{aligned}
$$

It is clear that inequality (8) holds for all integers $k \geq 5$, so inequality (7) is true. Now, applying (7) repeatedly, we have

$$
\begin{aligned}
\sum_{k=2 m}^{\infty} \frac{1}{P_{k}^{3}} & =\sum_{k=m}^{\infty}\left(\frac{1}{P_{2 k}^{3}}+\frac{1}{P_{2 k+1}^{3}}\right) \\
& >\frac{1}{P_{2 m}^{2} P_{2 m-1}+3 P_{2 m} P_{2 m-1}^{2}-\frac{1}{82}\left(61 P_{2 m}+91 P_{2 m-1}\right)+\frac{1}{82}}
\end{aligned}
$$

Combining (6) and (9), we may immediately deduce inequality (2).

Now we consider that $n=2 m+1$ is an odd number. It is clear that in this case our theorem is equivalent to

$$
\begin{aligned}
& P_{2 m+1}^{2} P_{2 m}+3 P_{2 m+1} P_{2 m}^{2}+\frac{1}{82}\left(61 P_{2 m+1}+91 P_{2 m}\right) \\
& \quad<\left(\sum_{k=2 m+1}^{\infty} \frac{1}{P_{k}^{3}}\right)^{-1}<P_{2 m+1}^{2} P_{2 m}+3 P_{2 m+1} P_{2 m}^{2}+\frac{1}{82}\left(61 P_{2 m+1}+91 P_{2 m}\right)+\frac{1}{82}
\end{aligned}
$$

or

$$
\begin{aligned}
& \frac{1}{P_{2 m+1}^{2} P_{2 m}+3 P_{2 m+1} P_{2 m}^{2}+\frac{1}{82}\left(61 P_{2 m+1}+91 P_{2 m}\right)+\frac{1}{82}} \\
& \quad<\sum_{k=2 m+1}^{\infty} \frac{1}{P_{k}^{3}}<\frac{1}{P_{2 m+1}^{2} P_{2 m}+3 P_{2 m+1} P_{2 m}^{2}+\frac{1}{82}\left(61 P_{2 m+1}+91 P_{2 m}\right)} .
\end{aligned}
$$

First we prove the inequality

$$
\begin{aligned}
\frac{1}{P_{2 k+1}^{3}}+\frac{1}{P_{2 k+2}^{3}}< & \frac{1}{P_{2 k+1}^{2} P_{2 k}+3 P_{2 k+1} P_{2 k}^{2}+\frac{1}{82}\left(61 P_{2 k+1}+91 P_{2 k}\right)} \\
& -\frac{1}{P_{2 k+1}^{2} P_{2 k}+3 P_{2 k+1} P_{2 k}^{2}+\frac{1}{82}\left(61 P_{2 k+1}+91 P_{2 k}\right)}
\end{aligned}
$$

It is easy to check that inequality (11) is correct for $k=1,2$ and 3 . So, we can assume that $k \geq 4$. Note that $P_{2 k+1}^{3}=\frac{1}{8}\left(P_{6 k+3}+3 P_{2 k+1}\right), P_{2 k+2}^{3}=\frac{1}{8}\left(P_{6 k+6}-3 P_{2 k+2}\right), P_{2 k+1}^{3}+P_{2 k+2}^{3}=$ $\frac{1}{8}\left(P_{6 k+6}+P_{6 k+3}+3 P_{2 k+1}-3 P_{2 k+2}\right), P_{2 k+1}^{3} P_{2 k+2}^{3}=\frac{1}{512}\left(Q_{12 k+9}+6 Q_{8 k+6}+9 Q_{4 k+3}-4\right), P_{2 k+1}^{2} P_{2 k}+$ 
$3 P_{2 k+1} P_{2 k}^{2}=\frac{1}{8}\left(P_{6 k+2}+3 P_{6 k+1}-5 P_{2 k+1}-5 P_{2 k}\right)$, so inequality (11) is equivalent to the inequality

$$
\begin{aligned}
& \frac{P_{6 k+6}+P_{6 k+3}+3 P_{2 k+1}-3 P_{2 k+2}}{Q_{12 k+9}+6 Q_{8 k+6}+9 Q_{4 k+3}-4} \\
& \quad<\frac{378 P_{6 k+2}+154 P_{6 k+1}+\frac{78}{41} P_{2 k+2}+\frac{318}{41} P_{2 k+1}}{\left(P_{6 k+2}+3 P_{6 k+1}+\frac{39}{41} P_{2 k+1}+\frac{159}{41} P_{2 k}\right)\left(P_{6 k+8}+3 P_{6 k+7}+\frac{39}{41} P_{2 k+3}+\frac{159}{41} P_{2 k+2}\right)}
\end{aligned}
$$

From the definition and properties of the Pell-Lucas numbers, we have

$$
\left(P_{6 k+2}+3 P_{6 k+1}\right)\left(P_{6 k+8}+3 P_{6 k+7}\right)=\left(8 Q_{12 k+9}+10 Q_{12 k+8}+2,772\right) / 8
$$

and

$$
\begin{aligned}
\left(P_{6 k+2}+3 P_{6 k+1}+\frac{39}{41} P_{2 k+1}+\frac{159}{41} P_{2 k}\right)\left(P_{6 k+8}+3 P_{6 k+7}+\frac{39}{41} P_{2 k+3}+\frac{159}{41} P_{2 k+2}\right) \\
=\frac{1}{8}\left(8 Q_{12 k+9}+10 Q_{12 k+8}+\frac{7,344}{41} Q_{8 k+5}+\frac{2,124}{41} Q_{8 k+4}-\frac{31,844,565}{1,681} Q_{4 k-1}\right. \\
\left.\quad-\frac{13,186,923}{1,681} Q_{4 k-2}+\frac{4,442,760}{1,681}\right) .
\end{aligned}
$$

By these two identities and (12), we deduce that inequality (11) is equivalent to

$$
\begin{aligned}
& \frac{P_{6 k+6}+P_{6 k+3}+3 P_{2 k+1}-3 P_{2 k+2}}{Q_{12 k+9}+6 Q_{8 k+6}+9 Q_{4 k+3}-4} \\
& \quad<\frac{378 P_{6 k+2}+154 P_{6 k+1}+\frac{78}{41} P_{2 k+2}+\frac{318}{41} P_{2 k+1}}{8 Q_{12 k+9}+10 Q_{12 k+8}+\frac{7,344}{41} Q_{8 k+5}+\frac{2,124}{41} Q_{8 k+4}-\frac{31,844,565}{1,681} Q_{4 k-1}-\frac{13,186,923}{1,681} Q_{4 k-2}+\frac{4,442,760}{1,681}}
\end{aligned}
$$

For convenience, we let

$$
\begin{aligned}
A^{\prime}= & \left(8 Q_{12 k+9}+10 Q_{12 k+8}+\frac{7,344}{41} Q_{8 k+5}+\frac{2,124}{41} Q_{8 k+4}-\frac{31,844,565}{1,681} Q_{4 k-1}\right. \\
& \left.-\frac{13,186,923}{1,681} Q_{4 k-2}+\frac{4,442,760}{1,681}\right) \times\left(P_{6 k+6}+P_{6 k+3}+3 P_{2 k+1}-3 P_{2 k+2}\right)
\end{aligned}
$$

and $B^{\prime}=\left(Q_{12 k+9}+6 Q_{8 k+6}+9 Q_{4 k+3}-4\right)\left(378 P_{6 k+2}+154 P_{6 k+1}+\frac{78}{41} P_{2 k+2}+\frac{318}{41} P_{2 k+1}\right)$. Then we have

$$
\begin{aligned}
A^{\prime}= & 8\left(P_{18 k+15}-P_{6 k+3}\right)+10\left(P_{18 k+14}-P_{6 k+2}\right)+\frac{7,344}{41}\left(P_{14 k+11}-P_{2 k-1}\right) \\
& +\frac{2,124}{41}\left(P_{14 k+10}-P_{2 k-2}\right)-\frac{31,844,565}{1,681}\left(P_{10 k+5}-P_{2 k+7}\right)-\frac{13,186,923}{1,681}\left(P_{10 k+4}\right. \\
& \left.+P_{2 k+8}\right)+\frac{4,442,760}{1,681} P_{6 k+6}+8\left(P_{18 k+12}+P_{6 k+6}\right)+10\left(P_{18 k+11}+P_{6 k+5}\right) \\
& +\frac{7,344}{41}\left(P_{14 k+8}+P_{2 k+2}\right)+\frac{2,124}{41}\left(P_{14 k+7}+P_{2 k+1}\right)-\frac{31,844,565}{1,681}\left(P_{10 k+2}-P_{2 k+4}\right) \\
& -\frac{13,186,923}{1,681}\left(P_{10 k+1}+P_{2 k+5}\right)+\frac{4,442,760}{1,681} P_{6 k+3}+3 \times 8\left(P_{14 k+10}+P_{10 k+8}\right)
\end{aligned}
$$




$$
\begin{aligned}
& +3 \times 10\left(P_{14 k+9}+P_{10 k+7}\right)+\frac{7,344 \times 3}{41}\left(P_{10 k+6}+P_{6 k+4}\right)+\frac{2,124 \times 3}{41}\left(P_{10 k+5}\right. \\
& \left.+P_{6 k+3}\right)-\frac{31,844,565 \times 3}{1,681}\left(P_{6 k}+P_{2 k-2}\right)-\frac{13,186,923 \times 3}{1,681}\left(P_{6 k-1}+P_{2 k-3}\right) \\
& +\frac{4,442,760 \times 3}{1,681} P_{2 k+1}-3 \times 8\left(P_{14 k+11}-P_{10 k+7}\right)-3 \times 10\left(P_{14 k+10}-P_{10 k+6}\right) \\
& -\frac{7,344 \times 3}{41}\left(P_{10 k+7}-P_{6 k+3}\right)-\frac{2,124 \times 3}{41}\left(P_{10 k+6}-P_{6 k+2}\right)+\frac{31,844,565 \times 3}{1,681}\left(P_{6 k+1}\right. \\
& \left.-P_{2 k-3}\right)+\frac{13,186,923 \times 3}{1,681}\left(P_{6 k}-P_{2 k-4}\right)-\frac{4,442,760 \times 3}{1,681} P_{2 k+2} \\
& =154 P_{18 k+12}+70 P_{18 k+11}+\frac{95,514}{41} P_{14 k+8}+\frac{38,910}{41} P_{14 k+7}-\frac{506,756,250}{1,681} P_{10 k+2} \\
& -\frac{209,906,976}{1,681} P_{10 k+1}+\frac{983,915,086}{1,681} P_{6 k} \\
& +\frac{407,692,984}{1,681} P_{6 k-1}-\frac{771,966,210}{1,681} P_{2 k-3} \\
& -\frac{304,496,118}{1,681} P_{2 k-4} \\
& B^{\prime}=378\left(P_{18 k+11}-P_{6 k+7}\right)+154\left(P_{18 k+10}+P_{6 k+8}\right)+\frac{78}{41}\left(P_{14 k+11}-P_{10 k+7}\right) \\
& +\frac{318}{41}\left(P_{14 k+10}+P_{10 k+8}\right)+6 \times 378\left(P_{14 k+8}-P_{2 k+4}\right)+924\left(P_{14 k+7}+P_{2 k+5}\right) \\
& +\frac{468}{41}\left(P_{10 k+8}-P_{6 k+4}\right)+\frac{1,908}{41}\left(P_{10 k+7}+P_{6 k+5}\right)+9 \times 378\left(P_{10 k+5}-P_{2 k-1}\right) \\
& +1,386\left(P_{10 k+4}-P_{2 k-2}\right)+\frac{702}{41}\left(P_{6 k+5}-P_{2 k+1}\right)+\frac{318 \times 9}{41}\left(P_{6 k+4}+P_{2 k+2}\right) \\
& -4 \times 378 P_{6 k+2}-4 \times 154 P_{6 k+1}-\frac{78 \times 4}{41} P_{2 k+2}-\frac{318 \times 4}{41} P_{2 k+1} \\
& =154 P_{18 k+12}+70 P_{18 k+11}+\frac{95,514}{41} P_{14 k+8}+\frac{38,910}{41} P_{14 k+7}+\frac{158,064}{41} P_{10 k+5} \\
& +\frac{64,416}{41} P_{10 k+4}-\frac{36,496}{41} P_{6 k+2}-\frac{14,880}{41} P_{6 k+1}-\frac{225,516}{41} P_{2 k-1}-\frac{92,796}{41} P_{2 k-2} \text {. }
\end{aligned}
$$

Note that $P_{n+2}=2 P_{n+1}+P_{n}$, we have

$$
\begin{aligned}
B^{\prime}-A^{\prime}= & \frac{597,729,018}{1,681} P_{10 k+2}+\frac{247,592,208}{1,681} P_{10 k+1}-\frac{992,616,926}{1,681} P_{6 k} \\
& -\frac{411,295,736}{1,681} P_{6 k-1}+\frac{718,126,158}{1,681} P_{2 k-3}+\frac{282,199,170}{1,681} P_{2 k-4} \\
= & \left(\frac{3,483,829,506}{1,681} P_{10 k}-\frac{992,616,926}{1,681} P_{6 k}\right)+\left(\frac{1,443,050,244}{1,681} P_{10 k-1}\right. \\
& \left.-\frac{411,295,736}{1,681} P_{6 k-1}\right)+\frac{718,126,158}{1,681} P_{2 k-3}+\frac{282,199,170}{1,681} P_{2 k-4}>0
\end{aligned}
$$

for all integers $k \geq 4$. So, inequalities (11), (12) and (13) hold for all integers $k \geq 4$. 
Now, applying (11) repeatedly, we have

$$
\begin{aligned}
\sum_{k=2 m+1}^{\infty} \frac{1}{P_{k}^{3}}= & \sum_{k=m}^{\infty}\left(\frac{1}{P_{2 k+1}^{3}}+\frac{1}{P_{2 k+2}^{3}}\right) \\
& <\sum_{k=m}^{\infty} \frac{1}{P_{2 k+1}^{2} P_{2 k}+3 P_{2 k+1} P_{2 k}^{2}+\frac{1}{82}\left(61 P_{2 k+1}+91 P_{2 k}\right)} \\
& -\sum_{k=m}^{\infty} \frac{1}{P_{2 k+3}^{2} P_{2 k+2}+3 P_{2 k+3} P_{2 k+2}^{2}+\frac{1}{82}\left(61 P_{2 k+3}+91 P_{2 k+2}\right)}
\end{aligned}
$$

On the other hand, we prove the inequality

$$
\begin{aligned}
\frac{1}{P_{2 k+1}^{3}}+\frac{1}{P_{2 k+2}^{3}}> & \frac{1}{P_{2 k+1}^{2} P_{2 k}+3 P_{2 k+1} P_{2 k}^{2}+\frac{1}{82}\left(61 P_{2 k+1}+91 P_{2 k}\right)+\frac{1}{82}} \\
& -\frac{1}{P_{2 k+3}^{2} P_{2 k+2}+3 P_{2 k+3} P_{2 k+2}^{2}+\frac{1}{82}\left(61 P_{2 k+3}+91 P_{2 k+2}\right)+\frac{1}{82}}
\end{aligned}
$$

It is easy to check that inequality (15) is correct for $k=1,2$ and 3 . So, we can assume that $k \geq 4$. This time, inequality (15) is equivalent to

$$
\begin{aligned}
& \frac{P_{6 k+6}+P_{6 k+3}+3 P_{2 k+1}-3 P_{2 k+2}}{Q_{12 k+9}+6 Q_{8 k+6}+9 Q_{4 k+3}-4} \\
& \quad>\frac{378 P_{6 k+2}+154 P_{6 k+1}+\frac{78}{41} P_{2 k+2}+\frac{318}{41} P_{2 k+1}}{\left(P_{6 k+2}+3 P_{6 k+1}+\frac{39}{41} P_{2 k+1}+\frac{159}{41} P_{2 k}+\frac{4}{41}\right)\left(P_{6 k+8}+3 P_{6 k+7}+\frac{39}{41} P_{2 k+3}+\frac{159}{41} P_{2 k+2}+\frac{4}{41}\right)}
\end{aligned}
$$

or

$$
\begin{aligned}
& \frac{4}{41}\left(P_{6 k+6}+P_{6 k+3}+3 P_{2 k+1}-3 P_{2 k+2}\right)\left(60 P_{6 k+4}+40 P_{6 k+3}+\frac{552}{41} P_{2 k+1}+\frac{396}{41} P_{2 k}+\frac{4}{41}\right) \\
& \quad>B^{\prime}-A^{\prime}
\end{aligned}
$$

or

$$
\begin{aligned}
& 140 Q_{12 k+9}+140 Q_{12 k+8}-\frac{17,904}{41} Q_{8 k+4}-\frac{8,352}{41} Q_{8 k+3}+\frac{19,116}{41} Q_{4 k+2}+\frac{9,444}{41} Q_{4 k+1} \\
& +\frac{4}{41} P_{6 k+6}+\frac{4}{41} P_{6 k+3}+\frac{12}{41} P_{2 k+1}-\frac{12}{41} P_{2 k+2}+\frac{21,152}{41}>\frac{41}{4}\left(B^{\prime}-A^{\prime}\right) .
\end{aligned}
$$

It is clear that inequality (16) holds for all integers $k \geq 4$, so inequality (15) is true. Now, applying (15) repeatedly, we have

$$
\begin{aligned}
\sum_{k=2 m+1}^{\infty} \frac{1}{P_{k}^{3}} & =\sum_{k=m}^{\infty}\left(\frac{1}{P_{2 k+1}^{3}}+\frac{1}{P_{2 k+2}^{3}}\right) \\
& >\frac{1}{P_{2 m+1}^{2} P_{2 m}+3 P_{2 m+1} P_{2 m}^{2}+\frac{1}{82}\left(61 P_{2 m+1}+91 P_{2 m}\right)+\frac{1}{82}}
\end{aligned}
$$

Combining (14) and (17), we may immediately deduce inequality (10). 
Now our theorem follows from inequalities (2) and (10). This completes the proof of our theorem.

\section{Competing interests}

The authors declare that they have no competing interests.

\section{Authors' contributions}

ZX drafted the manuscript. TW participated in its design and coordination and helped to draft the manuscript. All authors read and approved the final manuscript.

\section{Acknowledgements}

The authors express their gratitude to the referee for his very helpful and detailed comments. This work is supported by the N.S.F. $(11001218,11071194)$ of P.R. China and the Research Fund for the Doctoral Program of Higher Education (20106101120001) of P.R. China and the G.I.C.F. (YZZ12065) of NWU.

Received: 17 May 2013 Accepted: 4 June 2013 Published: 26 June 2013

\section{References}

1. Kilic, E, Altunkaynak, B, Tasci, D: On the computing of the generalized order-k Pell numbers in log time. Appl. Math. Comput. 181, 511-515 (2006)

2. Hao, P: Arithmetic properties of q-Fibonacci numbers and q-Pell numbers. Discrete Math. 306, $2118-2127$ (2006)

3. Santos, JPO, Sills, AV: q-Pell sequences and two identities of V.A. Lebesgue. Discrete Math. 257, 125-142 (2002)

4. Kilic, E: The generalized order-k Fibonacci-Pell sequences by matrix methods. J. Comput. Appl. Math. 209, 133-145 (2007)

5. Egge, ES, Mansour, T: 132-avoiding two-stack sortable permutations, Fibonacci numbers, and Pell numbers. Discrete Appl. Math. 143, 72-83 (2004)

6. Mansour, T, Shattuck, M: Restricted partitions and q-Pell numbers. Cent. Eur. J. Math. 9, 346-355 (2011)

7. Zhang, W, Wang, T: The infinite sum of reciprocal Pell numbers. Appl. Math. Comput. 218, 6164-6167 (2012)

8. Zhang, W, Wang, T: The infinite sum of reciprocal of the square of the Pell numbers. J. Weinan Teach. Univ. 26, 39-42 (2011)

9. Ohtsuka, H, Nakamura, S: On the sum of reciprocal Fibonacci numbers. Fibonacci Q. 46/47, 153-159 (2008/2009)

10. Komatsu, T, Laohakosol, V: On the sum of reciprocals of numbers satisfying a recurrence relation of order $\mathrm{s}$. J. Integer Seq. 13, Article ID 10.5.8 (2010)

doi:10.1186/1687-1847-2013-184

Cite this article as: Xu and Wang: The infinite sum of the cubes of reciprocal Pell numbers. Advances in Difference

Equations 2013 2013:184.

\section{Submit your manuscript to a SpringerOpen ${ }^{\ominus}$ journal and benefit from:}

- Convenient online submission

Rigorous peer review

- Immediate publication on acceptance

Open access: articles freely available online

- High visibility within the field

- Retaining the copyright to your article 